\title{
Why should we expect a structure-modifying osteoarthritis drug to relieve osteoarthritis pain?
}

\section{Kenneth D Brandt}

In patients with knee osteoarthritis (OA) the real problem, for the clinician and the patient, is not radiographic OA but painful $\mathrm{OA}$. As noted by Liang, 'x-rays don't weep'. ${ }^{1}$ Patients weep.

In this issue of the Annals, Snijders et $a^{2}$ report results of a randomised controlled (RCT), placebo-controlled trial of doxycycline (doxy) in patients with symptomatic knee OA. Their primary end point was the difference in the proportion of subjects in the two treatment groups who achieved a clinical response at 24 weeks, as judged by OMERACTOARSI responder criteria. Secondary outcomes included joint pain, stiffness, function, quality of life, OA-related medication use and side effects of treatment. The results showed no difference between doxy and placebo with respect to symptomatic benefit; about one-third of each treatment group met the responder criteria. The authors conclude that doxy is ineffective in reducing symptoms of knee OA over 24 weeks.

The study was prompted by our 2005 report that doxy slowed the rate of joint space narrowing (JSN) over 30 months in patients with knee OA; significant differences from placebo were seen as early as 16 months. ${ }^{3}$ In contrast to the study by Snijders et al, ${ }^{2}$ our primary outcome measure was the effect of doxy on radiographic progression of $\mathrm{OA}$, not on symptoms. Specifically, our intent was to ascertain whether a fluoroscopically assisted semiflexed radiographic view of the $\mathrm{knee}^{4}$ was suitable for use in a multicentre clinical trial of a putative structure-modifying OA drug (SMOAD). Subjects enrolled in the study, which was implemented in 1997, were recruited chiefly from the community, rather than from clinics for patients with joint pain. Our inclusion criteria did not contain a threshold for severity of joint pain and levels of knee pain at entry were low.

\section{Correspondence to}

Kenneth D Brandt, Clinical Professor of Medicine, University of Kansas Medical Center, Kansas City, 5755 Windsor Drive, Fairway, Kansas USA 66205; kenbrandt@yahoo.com
Post hoc comparison showed that a smaller proportion of subjects in the doxy group reported $>20 \%$ increase in their knee pain score than in the placebo group, but that has not been validated as an outcome measure.

Until the current report by Snijders et $a{ }^{2}{ }^{2}$ no one had reported a RCT that examined the clinical relevance of the effect of doxy in slowing JSN in OA knees. For this reason, and because of the existence of ongoing RCTs of putative SMOADs, for example, salmon calcitonin ${ }^{5}$ and an inhibitor of inducible nitric oxide synthase, ${ }^{6}$ the findings of Snijders et $a l^{2}$ prompt a relevant question: 'Is pharmacological retardation of articular cartilage loss in patients with OA accompanied by significant symptomatic benefit?'.

Would the patient with OA pain 'weep' less if she were to take a 'chondroprotective' drug? If so, why? Articular cartilage is generally considered to be aneural, ${ }^{7}$ that is, not to be a tissue from which joint pain originates. It is a limitation of the study by Snijders et al that it reports the absence of a beneficial effect of doxy on OA symptoms in patients in whom an effect of the drug on JSN was not assessed, as it was in the earlier study. ${ }^{3}$ Although the demonstration of an absence of an effect of doxy on symptoms adds importantly to our knowledge about the clinical efficacy of doxy in patients with knee OA, it neither confirms nor refutes the previous report of a SMOAD effect of doxy.

Since implementation of our doxy study, understanding of $O A$ has grown: it is now clear, for example, that OA is not merely a cartilage disease but a disorder that affects all tissues of the joint and can have its origin in any of them; that common, garden-variety $\mathrm{OA}$ has a mechanical aetiopathogenesis; and that, although pain in OA joints may be due, for example, to periarticular bursitis, enthesopathy, ligament strain or muscle spasm, it is often secondary to synovitis, medullary hypertension in the cancellous subchondral bone or bone marrow lesions (BMLs). As discussed below, each of these is a consequence of existing mechanically induced joint damage or its repair. ${ }^{8}$

\section{SYNOVITIS AS A CAUSE OF OA PAIN}

In some patients with advanced $O A$, the secondary synovitis may be so prominent as to suggest rheumatoid arthritis (RA). In contrast to RA, however, synovial cells in OA do not erode the cartilage at the joint margins. Fragments of fibrillated articular cartilage and, less commonly, of bone, that have broken off the articular surface, and soluble breakdown products from these tissues (eg, proteoglycans, collagen), may be incorporated into the synovial membrane, where they incite a chronic inflammatory response. How long these foci of detritus remain in the synovium, and whether a SMOAD would shorten that duration, are unknown. Furthermore, although reduction in synovitis may decrease symptoms in patients with OA, it did not slow progression of cartilage damage. ${ }^{9}$ Lack of correlation between the severity of synovitis and the severity of knee pain at baseline, and only modest correlation between the change in synovitis and the change in pain, also suggest that factors other than synovitis are important as a cause of OA pain. ${ }^{9}$

\section{BONE ANGINA AS A CAUSE OF OA PAIN}

In some patients with $\mathrm{OA}$, joint pain reflects 'bone angina', due to stagnation of blood flow through thickened subchondral trabeculae that have resulted from the healing and remodelling of microfractures. ${ }^{10}$ In hip OA, this may strikingly prolong the emptying time after intraosseous injection of radiopaque contrast material into the femoral neck. ${ }^{11}$ Normalisation of these changes by osteotomy promptly alleviates joint pain in these patients. In those who have undergone a hip or knee osteotomy, a procedure that relieves the stress on the load-bearing surface of the joint, long-term pain relief and functional improvement, and the reversal of structural damage, for example, JSN and subchondral sclerosis, may result. ${ }^{12} 13$

Evidence that the structural damage in an $\mathrm{OA}$ joint can be improved if the intra-articular stress is normalised by osteotomy is reinforced by evidence that unloading the $\mathrm{OA}$ joint by distraction can result in progressive and prolonged symptomatic and structural improvement. Among 22 patients who underwent distraction for severe ankle OA, clinical benefit persisted for at least 7 years 
in $73 \%$ and, on average, was sustained for 10 years. ${ }^{14}$ Marked and sustained improvement has been seen after distraction also in severe knee OA, ${ }^{15}$ although follow-up has not been as long as that in patients with ankle OA.

In patients with advanced posttraumatic ankle $\mathrm{OA}$, significant decreases in pain and improvement in function after distraction were accompanied by reduction in the density of subchondral bone in talus and tibia that persisted for at least 2 years of follow-up. The investigators suggested that more physiological distribution of stress by the less dense bone facilitated repair of articular cartilage. ${ }^{16}$ We suggest that it was related also to the observed symptomatic improvement. In a preliminary report ${ }^{17}$ of an uncontrolled study of patients with knee OA who were studied after distraction, changes in standardised radiography, MRI, and levels of biomarkers of synthesis and degradation of type II collagen all were consistent with cartilage repair.

Unloading the OA knee non-surgically, for example, with orthotics or bracing, may also have utility. For example, use of a lateral wedge orthotic slowed progression of medial compartment JSN over 3 years, relative to that which occurred with neutral orthotics, suggesting that improved biomechanics in the OA knee resulted in structural improvement. ${ }^{18}$ Similarly, a greater degree of toeing out during walking, which reduces the peak adductor moment at the knee, diminished the risk of medial JSN in OA knees. ${ }^{19}$

\section{BMLS AS A CAUSE OF OA PAIN}

In some patients with OA, BMLs may cause joint pain. In MRIs of OA knees, they are seen as focal areas of increased signal in the subchondral marrow in fatsuppressed T2-weighted images. They are consistent with the marrow lesions that McAlindon et al ${ }^{20}$ showed were strongly associated with isotope retention on a bone scan and that correlated strongly with subchondral sclerosis on the radiograph. Dieppe et $a^{21}$ showed that the bone scan had predictive value for radiographic progression of knee OA, as reflected by JSN. In scans that did not show focal areas of isotope retention, no progression was noted over a 5-year follow-up period. In contrast, among $\mathrm{OA}$ patients who had a 'hot' knee scan at baseline, progression was seen in approximately $50 \%$.

BMLs in patients with OA have erroneously been called bone marrow 'oedema' but histologically they are foci of fibrosis and osteonecrosis and bone remodelling, rather than accumulation of interstitial fluid. ${ }^{22}$ They are not specific for OA but may be seen with insufficiency fractures and a variety of other conditions. Hunter and colleagues ${ }^{23}$ have confirmed a link between elevated intraosseous venous pressure and BMLs.

In addition to their association with the progression of structural damage and joint pain in subjects with knee OA, BMLs have been associated with varus-valgus malalignment. ${ }^{24}$ Medial BMLs were seen mostly in varus limbs and lateral BMLs, mostly in valgus limbs. Furthermore, medial BMLs strongly predicted the risk of medial compartment JSN and lateral BMLs, of lateral compartment JSN. After adjustment for malalignment, the risk was reduced, but not eliminated. It has been suggested ${ }^{24}$ that progression of JSN may be due to the BMLs themselves, or that malalignment produced BMLs and loss of articular cartilage in the ipsilateral tibiofemoral compartment. We believe the latter is the more likely.

Osteotomy has now been largely superseded by arthroplasty as a surgical treatment for painful OA. In the hands of most orthopaedic surgeons, arthroplasty is a more reliable procedure and, for the patient, is followed by a much more rapid return to weight-bearing activity. Nonetheless, the earlier work on osteotomy and the more recent studies cited above that show that mechanical interventions that decrease loading of the knee can improve symptoms and structural damage underscore the aetiopathogenetic importance of abnormal joint mechanics in $\mathrm{OA}$.

In $\mathrm{OA}$, the severity and progression of symptoms correlate poorly with the severity and progression of structural damage. It has yet to be explained why, given comparable levels of pathology, some $\mathrm{OA}$ joints are painful and others are not. Understanding the aetiopathogenesis of OA pain may be much more relevant than understanding the mechanism(s) of cartilage breakdown in OA. Anxiety or depression, for example, may be better predictors of OA pain than the severity of radiographic change. ${ }^{25}$

The processes that are involved in attempts to contain the mechanical insult and heal the OA joint can be triggered by a variety of mechanical abnormalities but, in our opinion, without a return to mechanical normality, attempts at healing will fail. ${ }^{26}$ As long as the adverse mechanical environment that got the joint into trouble in the first place remains, it is unlikely that a pharmacological or biological agent that inhibits a specific enzyme or cytokine in the pathways of cartilage degradation or further stimulates the already increased synthesis of cartilage matrix molecules by chondrocytes will be effective. A recent post hoc analysis that found that varus angulation negated the effect of doxy in slowing $\mathrm{JSN}^{27}$ supports that view. On the other hand, if the excessive or aberrant loading of the joint is normalised, use of such agents may be superfluous. In our efforts to find new therapies for $\mathrm{OA}$, we have suggested that a search for the genes that control, for example, the shape of joints or neuromuscular coordination or that otherwise influence joint loading is more likely to be fruitful than a search for genes that regulate the synthesis or breakdown of articular cartilage. Furthermore, because normal subchondral bone is essential to contain the mechanical abnormalities that damage the overlying cartilage, emphasis on cartilage repair alone is unlikely to be met with therapeutic success. ${ }^{8}$

Given the causal relationships between abnormal loading of the OA joint, structural damage and joint pain and the failure of potential SMOADs to alter those relationships, it is not surprising that patients with symptomatic knee OA who were treated with doxy, even if it had been confirmed that doxy possessed SMOAD activity, did not 'weep' less than patients who received placebo. ${ }^{2}$ There is no reason to expect a SMOAD to modify the abnormal intra-articular stress that is the cause of common, garden variety $\mathrm{OA}$ and, secondarily, of OA pain.

Acknowledgements The author thanks his friends and colleagues, Paul Dieppe and Eric Radin, for their contributions to this discussions that helped formulate some of the contemporary concepts of OA discussed herein.

\section{Competing interest None}

Provenance and peer review Commissioned; externally peer reviewed.

Accecpted 24 April 2011 Ann Rheum Dis 2011;70:1175-1177

doi:10.1136/ard.2011.150953

\section{REFERENCES}

1. Liang MH. Pushing the limits of patient-oriented outcome measurements in the search for disease modifying treatments for osteoarthritis. J Rheumatol Supp/ 2004;70:61-5.

2. Snijders GF, van den Ende $\mathbf{C H}$, van Riel $\mathrm{P}$, et al The effects of doxycycline on reducing symptoms in knee osteoarthritis: results from a triple blinded randomized controlled trial. Ann Rheum Dis 2011;70:1191-96.

3. Brandt KD, Mazzuca SA, Katz BP, et al. Effect of doxycycline on progression of osteoarthritis: results of a randomized, placebo-controlled, double-blind trial. Arthritis Rheum 2005;52:2015-25. 
4. Buckland-Wright JC, Macfarlane DG Williams SA, et al. Accuracy and precision of joint space width measurements in standard and macroradiographs of osteoarthritic knees. Ann Rheum Dis 1995:54:872-80.

5. ClinicalTrials.gov. Efficacy and Safety of Oral Salmon Calcitonin in Patients with Knee Osteoarthritis (OA 2 study). http://clinicaltrials.gov/ ct2/show/NCT00704847 (accessed 11 May 2011).

6. ClinicalTrials.gov. A Long-Term, Placebo-Controlled $X$-Ray Study Investigating the Safety and Efficacy of SD-6010 in Subjects with Osteoarthritis of the Knee (ITIC). http://clinicaltrials.gov/ct2/show/ NCT00565812 (accessed 11 May 2011).

7. Meachim G, Stockwell RA. The matrix. In: Freeman MAR, ed. Adult Articular Cartilage. 2nd edition. Tunbridge Wells: Pitman Medical Publishing 1979:1-67.

8. Brandt KD, Dieppe P, Radin EL. Commentary: is it useful to subset 'primary' osteoarthritis? A critique based on evidence regarding the etiopathogenesis of osteoarthritis. Semin Arthritis Rheum 2009;39:81-95

9. Hill CL, Hunter DJ, Niu J, et al. Synovitis detected on magnetic resonance imaging and its relation to pain and cartilage loss in knee osteoarthritis. Ann Rheum Dis 2007;66:1599-603.

10. Radin EL. Osteoarthrosis - the orthopedic surgeon's perspective. Acta Orthop Scand Suppl 1995; 266:6-9

11. Arnoldi CC, Linderholm $H$, Müssbichler $H$. Venous engorgement and intraosseous hypertension in osteoarthritis of the hip. J Bone Joint Surg Br 1972;54:409-21.
12. Pauwels F. Biomechanics of the Normal and Diseased Hip: Theoretical Foundation, Technique and Results of Treatment. An Atlas. New York, NY: Springer Verlag 1976:129-268.

13. Maquet PG. Biomechanics of the Knee: With Application to the Pathogenesis and the Surgical Treatment of Osteoarthritis. 2nd edition. New York, NY: Springer Verlag 1984

14. Ploegmakers JJ, van Roermund PM, van Melkebeek J, et al. Prolonged clinical benefit from joint distraction in the treatment of ankle osteoarthritis. Osteoarthr Cartil 2005:13:582-8.

15. Intema F, Marijiissen ACA, Beekhuizen M, et al. Joint distraction in the treatment of severe knee osteoarthritis: the first results on clinical efficacy and structural repair. Ann Rheum Dis 2008;67(Suppl II):96

16. Intema F, Thomas TP, Anderson DD, et al. The clinical benefit of joint distraction in treatment of advanced ankle OA may be partly explained by subchondral bone changes. Arthritis Rheum 2010;62(Suppl):S74.

17. Intema F, Wiegant $K$, van Roermund PM, et al. Clinical improvement and structural tissue repair by joint distraction, in treatment of end-stage knee osteoarthritis. Arthritis Rheum 2010;62(Suppl):S574.

18. Gocker B, Demirag MD, Block JA. Lateral wedge orthotics delay progression of joint space narrowing in patients with medial knee osteoarthritis. Arthritis Rheum 2008;58(Suppl):S241.

19. Chang A, Hurwitz D, Dunlop D, et al. The relationship between toe-out angle during gait and progression of medial tibiofemoral osteoarthritis. Ann Rheum Dis 2007:66:1271-5.
20. McAlindon TE, Watt I, McCrae F, et al. Magnetic resonance imaging in osteoarthritis of the knee: correlation with radiographic and scintigraphic findings. Ann Rheum Dis 1991:50:14-19.

21. Dieppe $\mathbf{P}$, Cushnaghan J, Young P, et al. Prediction of the progression of joint space narrowing in osteoarthritis of the knee by bone scintigraphy. Ann Rheum Dis 1993;52:557-63.

22. Zanetti M, Bruder E, Romero J, et al. Bone marrow edema pattern in osteoarthritic knees: correlation between MR imaging and histologic findings. Radiology 2000;215:835-40.

23. Hunter DJ, Niu J, Zhang Y, et al. Altered perfusion and venous hypertension is present in regions of bone affected by BMLs in knee OA. Osteoarthritis Cartilage 2007;15(Suppl 3):C171-2.

24. Felson DT, McLaughlin S, Goggins J, et al. Bone marrow edema and its relation to progression of knee osteoarthritis. Ann Intern Med 2003:139:330-6.

25. Summers MN, Haley WE, Reveille JD, et al. Radiographic assessment and psychologic variables as predictors of pain and functional impairment in osteoarthritis of the knee or hip. Arthritis Rheum 1988; 31:204-9.

26. Brandt KD, Dieppe P, Radin EL. Etiopathogenesis of osteoarthritis. Rheum Dis Clin North Am 2008:34:531-59.

27. Mazzuca SA, Brandt KD, Chakr R, et al. Varus malalignment negates the structure-modifying benefits of doxycycline in obese women with knee osteoarthritis. Osteoarthr Cartil 2010;18:1008-11. 